\title{
Evidence for a current sheet forming in the wake of a coronal mass ejection from multi-viewpoint coronagraph observations ${ }^{\star}$
}

\author{
S. Patsourakos ${ }^{1}$ and A. Vourlidas ${ }^{2}$ \\ ${ }^{1}$ University of Ioannina, Department of Physics, Section of Astrogeophysics, Ioannina 45110, Greece \\ e-mail: spatsour@cc.uoi.gr \\ 2 Space Science Division, Naval Research Laboratory, Washington, DC 20375, USA \\ e-mail: vourlidas@nrl.navy.mil
}

Received 26 May 2010 / Accepted 1 October 2010

\begin{abstract}
Context. Ray-like features observed by coronagraphs in the wake of coronal mass ejections (CMEs) are sometimes interpreted as the white light counterparts of current sheets (CSs) produced by the eruption. The 3D geometry of these ray-like features is largely unknown and its knowledge should clarify their association to the CS and place constraints on CME physics and coronal conditions. Aims. If these rays are related to field relaxation behind CMEs, therefore representing current sheets, then they should be aligned to the CME axis. With this study we test these important implications for the first time.

Methods. An example of such a post-CME ray was observed by various coronagraphs, including these of the Sun Earth Connection Coronal and Heliospheric investigation (SECCHI) onboard the Solar Terrestrial Relations Observatory (STEREO) twin spacecraft and the Large Angle Spectrometric Coronagraph ( $L A S C O)$ onboard the Solar and Heliospheric Observatory $(\mathrm{SOHO})$. The ray was observed in the aftermath of a CME which occurred on 9 April 2008. The twin STEREO spacecraft were separated by about $48^{\circ}$ on that day. This significant separation combined with a third "eye" view supplied by LASCO allow for a truly multi-viewpoint observation of the ray and of the CME. We applied 3D forward geometrical modeling to the CME and to the ray as simultaneously viewed by SECCHI-A and B and by SECCHI-A and LASCO, respectively.

Results. We found that the ray can be approximated by a rectangular slab, nearly aligned with the CME axis, and much smaller than the $\mathrm{CME}$ in both terms of thickness and depth $\left(\approx 0.05\right.$ and $0.15 R_{\odot}$ respectively). The ray electron density and temperature were substantially higher than their values in the ambient corona. We found that the ray and CME are significantly displaced from the associated post-CME flaring loops.

Conclusions. The properties and location of the ray are fully consistent with the expectations of the standard CME theories for postCME current sheets. Therefore, our multi-viewpoint observations supply strong evidence that the observed post-CME ray is indeed related to a post-CME current sheet.
\end{abstract}

Key words. Sun: coronal mass ejections (CMEs) - Sun: flares

\section{Introduction}

CMEs result from a major restructuring of the corona. During such events, magnetic fields and plasma are expelled from the Sun causing dramatic changes in the coronal medium both within and around the erupting volume.

Essentially all CME models predict the formation of a postCME current sheet (CS) underneath the erupting magnetic flux rope (e.g. see also the review of Forbes \& Lin 2000). As the magnetic flux rope ${ }^{1}$ ascends, it pushes aside overlying magnetic fields. These fields will pinch off underneath the erupting rope forming a neutral CS, i.e. a surface separating fields of opposite magnetic polarity. The faster the eruption proceeds, the higher the rate at which new field lines accumulate at the CS. Such posteruption CSs should be distinguished from the CSs which could exist before the eruption in some CME models.

There exist several pieces of observational evidence supporting the existence of post-CME CSs both in the inner and in the outer corona. A major "smoking gun" for their existence is in

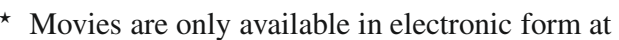
http://www. aanda.org

${ }^{1}$ Flux ropes are currently widely recognized as the major constituent of CMEs.
}

the form of white light (WL) rays seen by coronagraphs behind CMEs (e.g., Ko et al. 2003; Webb et al. 2003; Vršnak et al. 2009). The rays are formed in the wake of CMEs, they extend to several solar radii and persist from several hours up to few days. The detection of very hot $(>3 \mathrm{MK})$ plasmas in several of these WL rays by the UltraViolet Coronagraph Spectrometer (UVCS; Kohl et al. 1995) further supported the possibility that such structures represent CSs (e.g., Ko et al. 2003; Raymond et al. 2003; Bemporad et al. 2006; Lee et al. 2006; Lin et al. 2007). Coronal CSs are places where one could expect higher heating rates, and therefore higher temperatures. Moreover, such rays are not quiescent but they often exhibit dynamics such as propagating blobs through the ray (e.g., Sheeley \& Wang 2007). Such dynamic phenomena could result from reconnections taking place in a CS. A recent review of CS observations made by LASCO and UVCS is given in Vršnak et al. (2009). An analytical Petscheklike current sheet model was found in a good agreement with the observations. Closer to the solar surface, observations of cusplike hot structures and descending voids above eruption sites and arcades provided further support for the existence of post-CME CSs (e.g., McKenzie \& Hudson 1999; Innes et al. 2003; Asai et al. 2004; Sheeley et al. 2004). 
However there are a couple of reasons which argue against the possibility the observed rays could represent CSs. First, standard plasma theory predicts that CSs should be very narrow (a few meters or less) for typical coronal conditions (e.g., Litvinenko 1996; Wood \& Neukirch 2005). However, the coronagraph observations suggest CS widths at a fraction of the solar radius $0.2-0.8 R_{\odot}$ : Vršnak et al. 2009). One way to overcome this apparent contradiction is to invoke anomalous processes (e.g., turbulence, hyperesistivity) which could substantially broaden CSs (e.g., Lin et al. 2007; Bemporad 2008). Another possibility is that the macroscopic CS could be in reality the superposition of several small hot-spots, patches where small-scale reconnections take place (e.g., Klimchuk 1996; Cargill et al. 2006; Linton \& Longcope 2006; Onofri et al. 2006; Vršnak et al. 2009).

A second difficulty comes from the very nature of all past ray observations which employed a single view point. This does not allow to determine the full 3D geometry (i.e., width depth and height) of the rays and their exact relationship with the associated CMEs. All we could measure are quantities projected in the plane of sky (POS) of the corresponding instrument. Even more importantly one has to worry that projection effects in the optically thin corona could cause one to falsely believe that the observed rays represent post-eruption CSs. This is particularly true during CMEs which cause reconfigurations of significant coronal volumes in terms of rotations, deflections, displacements etc. of structures both within and around the CME. For instance the outer CME legs or structures around the erupting volume, could be falsely identified as candidate CSs when observed at a favorable angle; they may appear to trail the CME in projection as viewed from a single viewpoint. However, such structures may be far from the eruption core where the CS should lie.

Therefore we do not know the "true" width and depth of these rays, not to mention their shapes. Moreover, we currently ignore how they are arranged in 3D space with respect to the associated CME and whether they represent a projection effect. From the standard theory for the formation of post-CME CSs one would expect them to be quasi-aligned with the CME and to be bounded within it (i.e., to occupy a smaller volume than the $\mathrm{CME}$ itself). Clearly observations of post-CME rays from multiple and distinct viewpoints are required in order to address the above important questions.

The launch of the STEREO mission in late 2006 initiated an avenue for the study of the solar corona in $3 \mathrm{D}$. The mission consists of two almost identical spacecraft, one orbiting ahead (STA) and the other orbiting behind (STB) Earth's orbit. The two spacecraft drift apart at a rate of $\approx 45^{\circ}$ per year. With this paper we supply the first detailed 3D analysis of a post-CME ray using STEREO and LASCO data. Our analysis supplies some new and important geometrical and physical evidence that the observed ray could represent a CS.

Our paper is structured as follows. An overview of the CMEray observations is given in Sect. 2. Section 3 describes the $3 \mathrm{D}$ geometrical modeling applied to the $\mathrm{CME}$ and to the ray whereas in Sect. 4 we determine various physical parameters (density and temperature) of the ray. Finally, Sect. 5 summarizes our findings and discusses their implications.

\section{Overview of the observations}

We analyze here a CME event which took place on 9 April 2008. The event was initiated in the active region (AR) 1098 of the NOAA classification. There was no GOES flare associated with this CME: this could be due to the fact that the source active region was partially occulted behind the West limb as seen from the Earth. The event was observed by the Solar and Heliospheric Observatory (SOHO), Hinode, the Transition Region And Corona Explorer (TRACE) and STEREO. A synopsis of these observations was presented by Reeves et al. (2008), Landi et al. (2010) and Savage et al. (2010).

We have posted a movie (movie1.mpg) showing the low coronal development of the event. This movie consists of composite EUVI-195 ^ channel and COR1 extreme ultra-violet and WL images respectively taken by STA; a couple of representative snapshots from this movie is given in Fig. 1; the FESTIVAL software of Auchère et al. (2008) was used to generate the composite images.

The event started around 09:10 UT with the activation and the lift off of a prominence. The eruption moved initially parallel to the surface towards the southeast and was diverted outwards along a southwestern path. Such deflection may have been caused by the large equatorial coronal hole south of the host active region. The strongly non-radial propagation of the early CME can also be evidenced in the upper panel of Fig. 1 where we note a significant displacement between the axis of the emerging CME into the COR1 field of view (FOV) and the position angle of the host AR. The erupting prominence eventually gave rise to a typical 3-part CME (e.g., upper panel of Fig. 1). A rather faint linear feature emitting in soft Xrays in the trail of the erupting CME was observed by the X-Ray Telecope (XRT) on Hinode (Reeves et al. 2008; Savage et al. 2010). Combined analysis of the XRT and the EUV Imaging Telescope (EIS) Hinode observations showed that this feature had temperatures in the range $\approx 6-10 \mathrm{MK}$ (Landi et al. 2010). This ultra-hot linear structure in the wake of a CME may have been associated with a postCME CS. Indeed, XRT count-rates as predicted from a Petcheklike current sheet model were found in a good agreement with the weak signal of the mentioned above current-sheet like feature (Ko et al. 2010). Once the CME exited the COR1 FOV a WL ray was formed at its wake (e.g., lower panel of Fig. 1). Also Savage et al. (2010) observed with XRT mass flows (e.g., outflows, supra-arcade downflows) around the mentioned above faint linear feature which supplied evidence that this structure possibly represented a post-CME CS. We concentrate here on WL observations of the corona taken by the COR2 coronagraphs (FOV from 2.5-15 $R_{\odot}$ ) of the Sun-Earth Connection Coronal and Heliospheric Investigation (SECCHI; Howard et al. 2008) instrument suite on-board the twin STEREO spacecraft and by the $\mathrm{C} 2$ coronagraph (FOV from $2.5-6 R_{\odot}$ ) of the LASCO experiment on SOHO (Brueckner et al. 1995). On the day of our observations the twin STEREO spacecraft were separated by $\approx 48^{\circ}$. Our combined multi-viewpoint observations allowed to monitor and characterize in $3 \mathrm{D}$ the evolution and the structure of the $\mathrm{CME}$ and of the post-CME ray. Raw data were first treated with the standard data reduction routines. COR2 obtains both total WL brightness (TB) and polarized WL brightness (PB) images, whereas C2 normally obtains only TB images. Typical image cadence is of the order of 10-20 min. There were no UVCS observations of the ray as the instrument was observing lower in the corona (Landi et al. 2010).

Figure 2 contains representative TB images throughout the event for COR2 on STA (COR2A), C2 and COR2 on STB (COR2B) respectively; the full sequences can be found in the corresponding online movies (movie2.mpg, movie3.mpg and movie4.mpg). From each image in these figures and the associated movies a pre-event TB image was first subtracted creating a base-difference image sequence. Therefore, bright (dark) areas correspond to mass increase (decrease) respectively. 


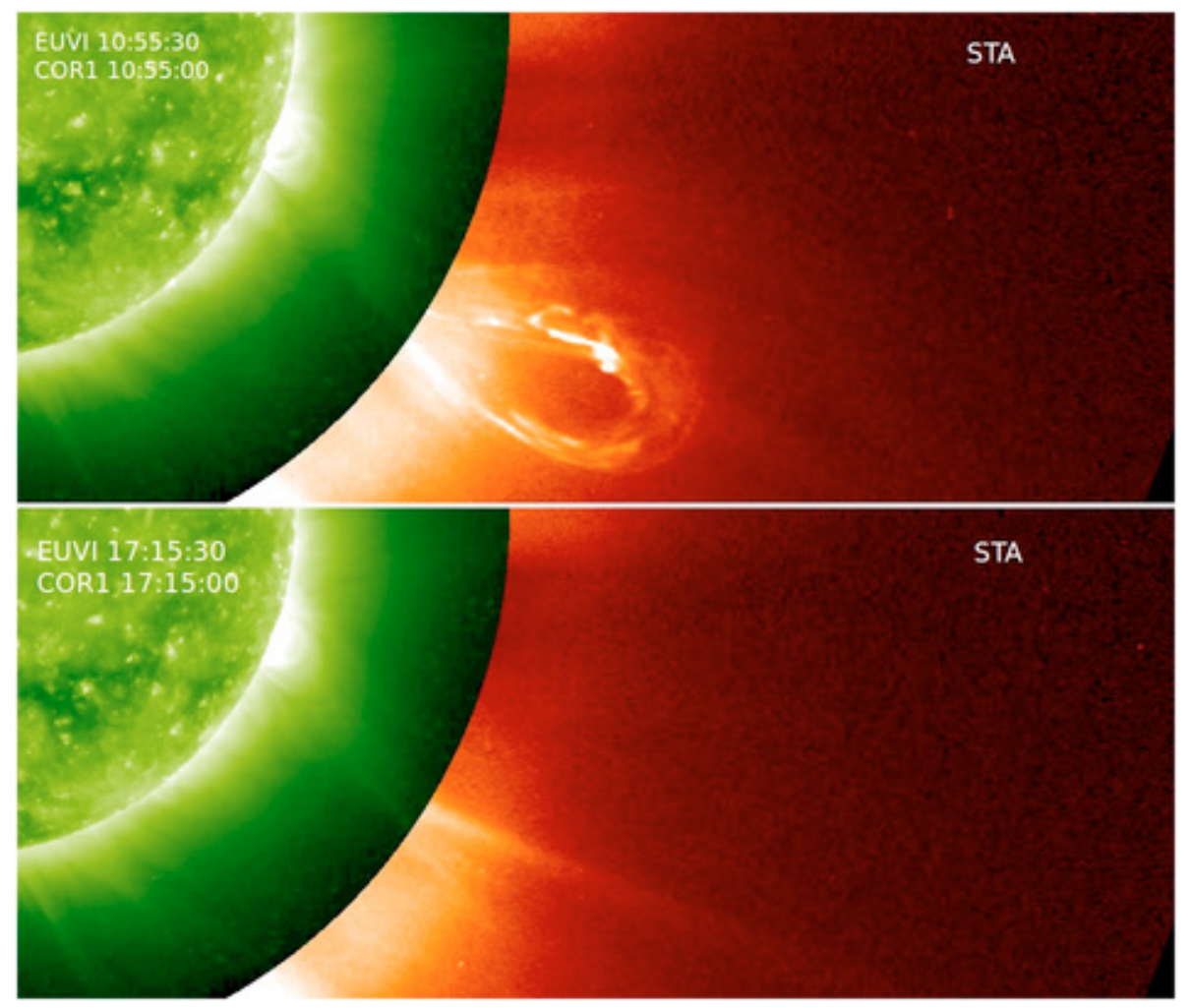

Fig. 1. Composite of EUVI-A 195 (inner FOV) and COR1-A (outer FOV) showing the emerging CME (upper panel) and the resulting WL ray (lower panel). The temporal evolution is shown by movie1.mpg.

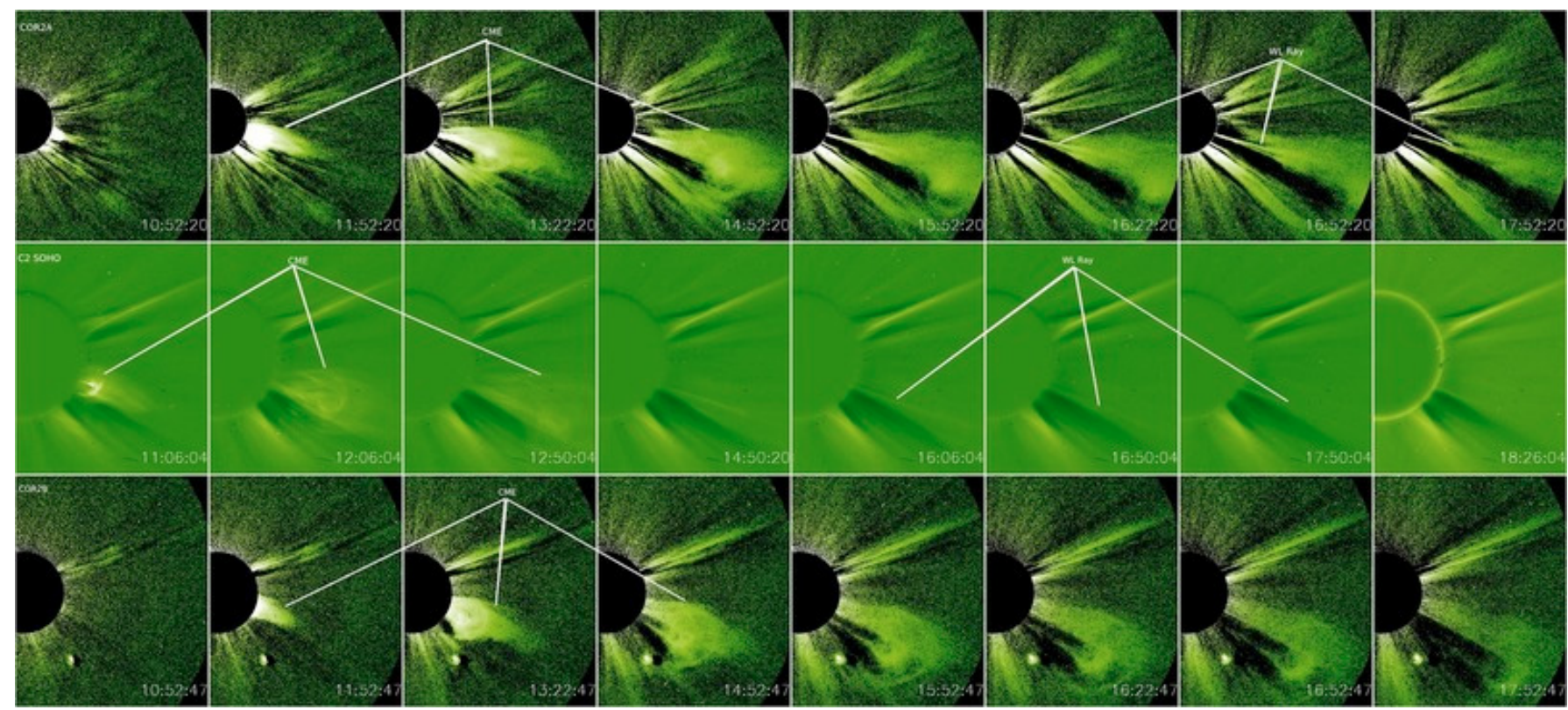

Fig. 2. Representative total brightness (TB) snapshots of the CME and of the WL ray as observed by COR2A, LASCO C2 and COR2B (upper, middle and lower panel respectively). Pre-event TB images taken at 01:27:13 UT (C2) and 09:22:00 UT (COR2A-B) were subtracted from each $\mathrm{C} 2$, COR2A, and COR2B frame. The C2 field of view spans 2.2-6 $R_{\odot}$ and the COR2A-B view spans $2.5-15 R_{\odot}$. The temporal evolution of the top, middle and bottom panels is shown in movie2.mpg, movie3.mpg and movie4.mpg, respectively.

Several remarks can now be made. The CME has already emerged into the COR2A and C2 FOV around 11:00; this happens later for COR2B and hints to a CME being closer to the STA and SOHO plane-of-sky (POS). Once it appears in the coronagraph FOVs the CME has the canonical 3-part CME structure with a bright front, dark cavity and bright core, i.e. it is a typical flux-rope CME. The CME core exhibits significant fine structure and consists of many threads as can be seen better in the $\mathrm{C} 2$ images. The CME seems to undergo a rotation as it expands.
Indeed, Thompson et al. (in prep.) studied the rotation of the erupting prominence that was associated with our CME. Using triangulations of several threads of the erupting prominence observed by STA and STB they determined its rotation profile for heliocentric distances $\approx 1.5-3.3 R_{\odot}$. They found that the prominence rotated by $\approx 120^{\circ}$ relative to its preeruption position. The CME starts to exit the SOHO FOV around 13:00 UT and the STA and STB FOVs around 15:52 UT and 16:52 UT, respectively. While this happens we see a WL ray forming in the wake 


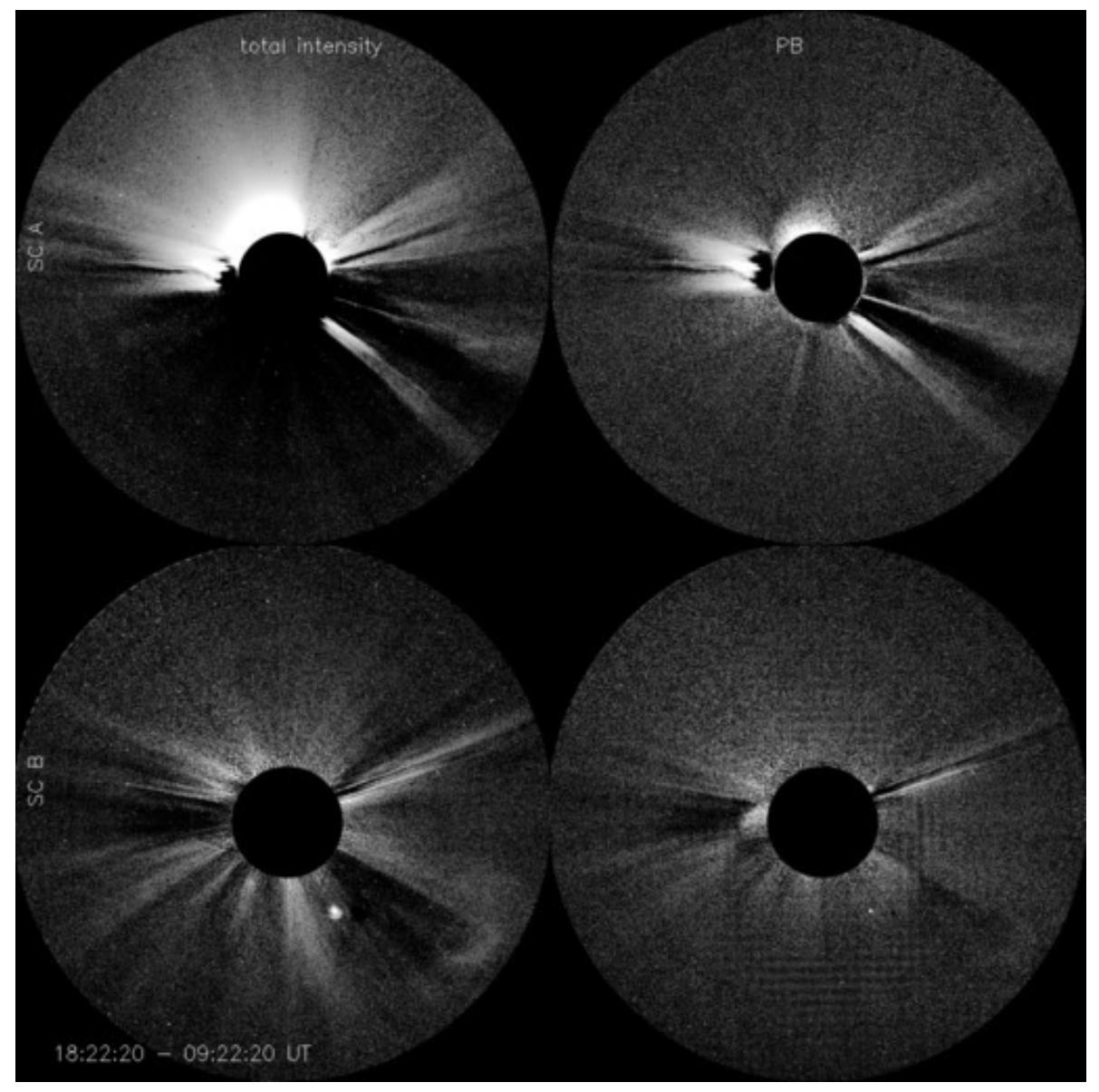

Fig. 3. TB and PB images for STA (first row) and for STB (second row). They were taken at 18:22:20 UT. Pre-event TB and PB images were subtracted. Field of view spans from $2.5-15 R_{\odot}$. of the CME in C2 (e.g., from 14:50 UT onwards in Fig. 2) and in COR2A (e.g., from 15:52 UT onwards in Fig. 2); there is no evidence for such a ray in STB though. The WL ray seems to undergo a rotation as the CME itself is rotating during its expansion. The WL ray connects to the bottom part of the CME cavity and seems to be formed when linear features joining the CME core start to pinch off as the CME propagates outwards. The ray was visible for at least one day after its formation.

From the above we conclude that the observed WL ray could be a candidate CS. One needs to always keep in mind that strictly speaking a CS is a surface separating magnetic fields of opposite direction: this is something which can not be determined by our observations or by any other similar observation given the lack of direct magnetic field observations in the corona. The 3D analysis of this paper will supply rather strong indirect evidence that the observed WL ray could have been a post-CME CS.

A first set of clues about the line of sight (LOS) extent of the post-CME ray and of the CME could be drawn from the analysis of the COR2 TB and PB images shown in Fig. 3. The theory of Thompson scattering (Billings 1966) which describes the formation of the WL emissions observed by coronagraphs predicts that $\mathrm{PB}$ decreases very fast when the emitting electrons lie away (i.e., $\approx \pm 20^{\circ}$ ) from the POS of a given instrument (Vourlidas \& Howard 2006). Therefore PB tends to emphasize structures close to a given POS; this is not the case for TB which has a more shallow fall-off with distance from the POS.

What we note from Fig. 3 is that the ray is absent in the STB PB and TB images whereas it can be seen in STA PB and TB. This means that the ray should lie close to the POS of STA.

Moreover, the LOS extent (depth) of the ray cannot be too large (i.e., comparable to the CME width). Otherwise, the ray would be visible in STB in either TB or PB since the CME itself was seen by both spacecraft in TB (Fig. 2) and in PB (Fig. 3; although less pronounced in STB). The above observations supply a rather strong indication that the ray could not have been due to a projection effect (e.g., widely separated CME legs lining up along the LOS) but it is rather a structure with limited extent along the LOS. This is further demonstrated and quantified in the next section.

\section{3D Geometrical Modeling of the CME and of the post-CME ray}

In order to determine the 3D structure of the post-CME ray and to infer its 3D position with respect to the CME we performed 3D geometrical modeling of both the CME and the ray.

We first start with the CME modeling. For this task we used the 3D forward model of Thernisien et al. (2006, 2009) (rtsccguicloud.pro and rtraytracewcs.pro ssw routines). The model has been successful in fitting the 3D morphology of CMEs (Thernisien et al. 2006, 2009) observed either from one viewpoint (LASCO) or from two viewpoints (STA and STB). The latter approach deemed particularly powerful in strongly constraining the CME parameters. We used TB images from COR2 on STA and STB.

Here, we model the observed CME with a spherical shell attached to two conical legs, a so-called "croissant" model. The spherical shell is meant to reproduce the upper part of the CME, whereas the conical legs are an approximation for the CME's lower sections, underneath the spherical shell. Such a model emulates the outline of a flux-rope, widely accepted as the magnetic structure of CMEs. The model is formulated in terms of the 
following free parameters: position (longitude $(\theta)$, latitude $(\phi)$ of the legs on the solar surface; tilt $(\gamma)$ of the model baseline with respect to the equator; height above the solar surface and aspect ratio (=height/radius) of the spherical shell. The model is radial, i.e. it lies vertical to the solar surface. A schematic of the model along with its free parameters can be found in Fig. 4 .

The model parameters were varied until we achieved a visually satisfactory agreement between the observed CME (in TB) and the wireframe projection of the model was achieved simultaneously for STA and STB (see left and right columns of Fig. 5 respectively). We note that the model reproduces fairly well both the body and the legs of the CME. We plot in the middle column of Fig. 5 synthetic TB images from the CME model for STA and STB to illustrate the model fitting further. A uniform density of $10^{6} \mathrm{~cm}^{-3}$ was assumed; its precise value does not affect the purely geometrical analysis of this section. Nevertheless the deduced geometrical information here will be used to determine the ray density in the following section. The figure shows that the model appears to reproduce some of the internal structure of the CME. The WL renderings from the model show some fine structure within the CME which is also present in the actual observations.

To estimate uncertainties in the model parameters we perturbed each parameter of the "best-fit" model shown in Fig. 5 by keeping the remaining parameters frozen. Each parameter was varied until a visually unacceptable solution (i.e., projections) was found; this supplied a measure of the uncertainty for each parameter. The fitting procedure was purely visual. Namely, we derived a set of parameters that give the best visual correspondance between the model and the two-viewpoint observations. The "best-fit" parameters along with their uncertainties of the CME model are given in Table 1 . We note that the uncertainties are quite small, which underlines the importance of using multi-view point data to tightly constrain the CME model. This is particularly true for the CME longitude which indicates the direction along which the CME propagates. Given the Carrington longitude of STA was $\approx 121^{\circ}$ on this day, the deduced Carrington longitude of the $\mathrm{CME}\left(\approx 194^{\circ}\right)$ implies that the $\mathrm{CME}$ was about $17^{\circ}$ away from the STA POS and therefore almost $11^{\circ}$ and $67^{\circ}$ degrees from the POS of SOHO and STB respectively. Therefore the CME was mainly an Earth and STA event. A schematic with the orientation and width of the fitted CME along with the locations of STB, Earth and STA and their corresponding POS can be found in Fig. 6.

We then performed 3D geometrical modeling of the COR2A and $\mathrm{C} 2$ ray observations. We used again the Thernisien et al. (2006, 2009) approach but with a different 3D geometrical model. A rectangular slab was used instead. This is a reasonable approximation for the ray shape since in both viewpoints it looks like an elongated with an almost constant cross-sectional area surface. The standard theory of solar eruptions (e.g., Forbes 2000) predicts post-CME current sheet shapes resembling rectangular slabs. Our ray model was formulated in terms of the longitude and latitude of the slab axis, and the slab width and depth. A schematic of the model along with its free parameters can be found in Fig. 4. Supposing for the moment that the ray represents a CS then its depth would correspond to the longitudinal extent of the post-CME arcade (along the erupting neutral line) whereas the ray width would correspond to the thickness of the current layer where opposing magnetic fields from both sides of the neutral line come into a diffusion region. For a ray viewed almost edge-on its depth will be comparable to the LOS extent. Since the ray width and depth are similar, the slab has a nearly square projection so the tilt becomes ambiguous (i.e., various

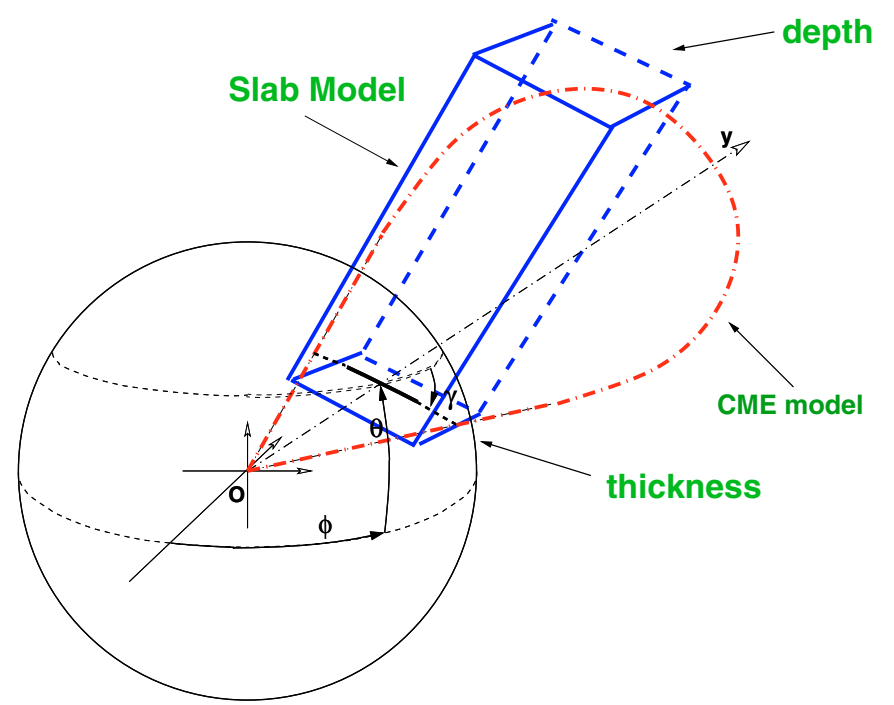

Fig. 4. Schematic of the 3D models used in the modeling of the WL CME (red) and ray (blue).

Table 1. CME and ray fitting results.

\begin{tabular}{ccc}
\hline \hline & CME & WL ray \\
\hline Carrington longitude $\left(^{\circ}\right)$ & $194 \pm 5$ & $197 \pm 5$ \\
Carrington latitude $\left(^{\circ}\right)$ & $-17 \pm 3$ & $-20 \pm 3$ \\
tilt $\left({ }^{\circ}\right)$ & $4 \pm 7$ & $4 \pm 7$ \\
aspect ratio & $0.16 \pm 0.03$ & $\mathrm{~N} / \mathrm{A}$ \\
angular width $\left(^{\circ}\right)$ & $18 \pm 4$ & $\mathrm{~N} / \mathrm{A}$ \\
depth $\left(R_{\odot}\right)$ & $\mathrm{N} / \mathrm{A}$ & $0.15 \pm 0.08$ \\
thickness $\left(R_{\odot}\right)$ & $\mathrm{N} / \mathrm{A}$ & $0.05 \pm 0.03$ \\
density $\left(\mathrm{cm}^{-3}\right)$ & $\mathrm{N} / \mathrm{A}$ & $3 \times 10^{7} \pm 5.3 \times 10^{6}$ \\
\hline
\end{tabular}

tilts can fit the data equally well). We therefore "fixed" the ray tilt to the "best-fit" tilt of the CME and derived a width and depth shown in Table 1.

Figure 7 contains our "best-fit" model of the ray. As done for the CME the "best-fit" model was determined by visual comparison between the model predictions and the actual multiviewpoint observations. As can be seen by this figure our model reproduces the ray orientation and projected size in both viewpoints. The uncertainties were derived the same way as done for the CME. The ray parameters along their uncertainties are given in Table 1. Again, the simultaneous fitting of the two viewpoints supplied strong constraints on the model parameters with the exception of the ray tilt angle due to its symmetry. For instance we note that increasing the ray depth and width by a factor of two we get unacceptable solutions (Fig. 8); the ray is too wide in the SOHO view. Two important remarks can be now made. First, the ray has a rather small width $\left(0.05 R_{\odot}\right)$ and depth $\left(0.15 R_{\odot}\right)$ which represent a small fraction of the solar radius. This can be contrasted with the large radius of the CME (e.g., around $1.8 R_{\odot}$ at 14:52 UT). Second, the location of the ray (i.e., longitude and latitude) is not very far off from the location of the CME. For instance the WL ray central longitude differs by only $3^{\circ}$ from that of the CME. This small difference is almost within the error bars and certainly much smaller than the CME width of $\approx 18^{\circ}$ This means that the ray is almost perfectly aligned with the CME and lies close to its center. Indeed the ratio between the CME to ray volumes is $\approx 50$, meaning that the ray represented only a small fraction of the CME volume. We will discuss the important implications of the above findings in Sect. 5. 


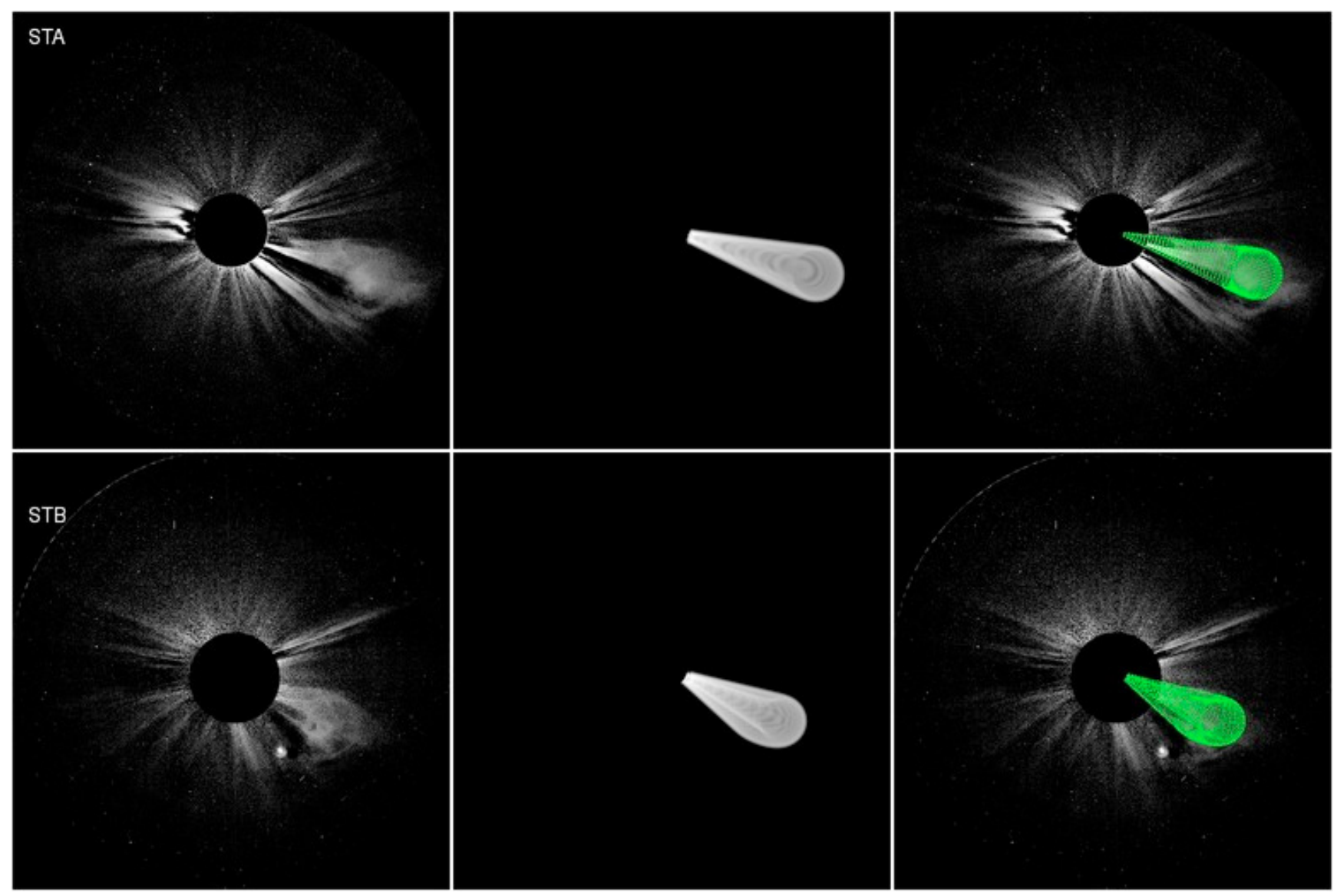

Fig. 5. 3D geometrical modeling of the CME observations of STA/COR2 (upper panel) and of STB/COR2 (lower panel). The left column shows the TB images for each spacecraft, where a pre-event image was first subtracted. The middle column shows synthetic TB images of the CME from our 3D modeling whereas the right column shows overlays of the CME wireframe(red) on the corresponding TB images. Observations taken at 14:52:20 UT for STA and at 14:52:47 for STB.

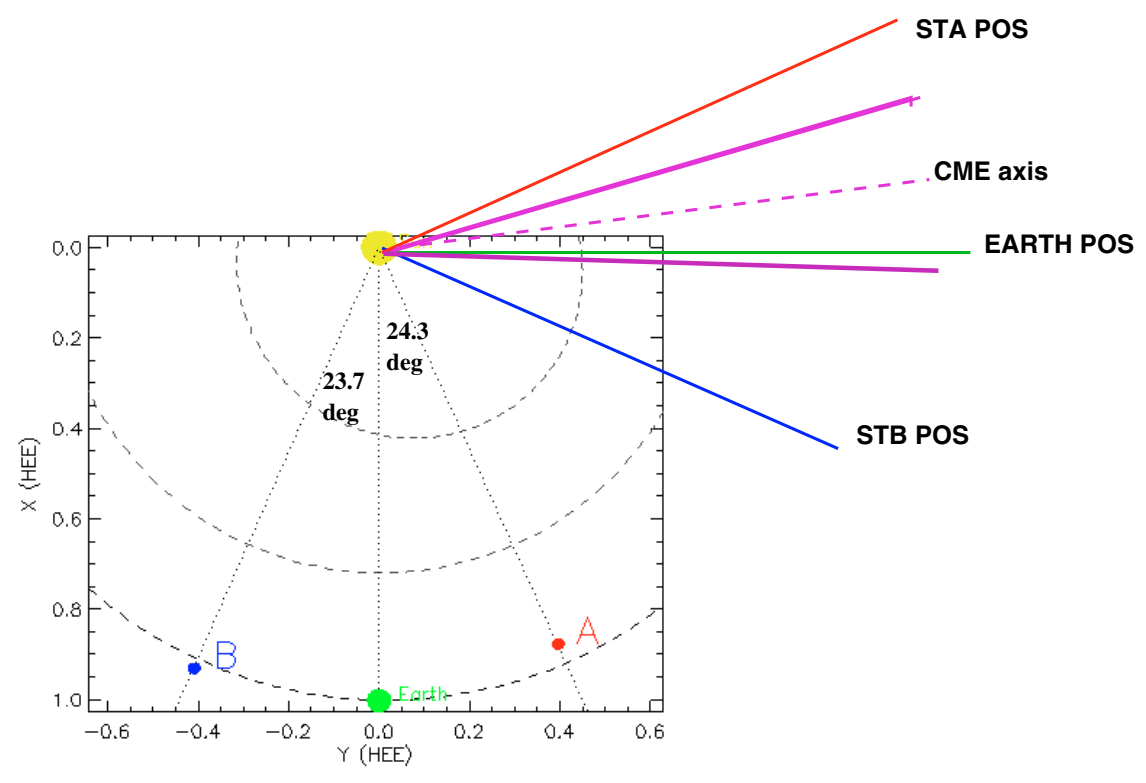

Fig. 6. Schematic of the orientation (dashed magenta line) and of the angular width of the CME (solid magenta lines) as determined from the 3D modeling of Sect. 3. The plot also supplies the relative positions of STB, Earth and ST as well as the corresponding POS (blue, green and red solid lines respectively) during 9 April 2008.

\section{Determination of the ray density structure}

Since the WL intensities in coronagraph images are proportional to the LOS integral of the electron density (e.g., Billings 1966) we used our observations to infer the density structure of the ray. The common assumption made in such determinations (e.g., Poland et al. 1981; Vourlidas et al. 2000; Vršnak et al. 2009) is that the structure of interest lies in the POS of the corresponding instrument. Then, local densities are inferred by assuming a LOS extent; a common assumption made is that it is proportional to the projected width of the structure.

Our measurements of the 3D shape and location of the ray allowed to relax the above two assumptions by supplying the 

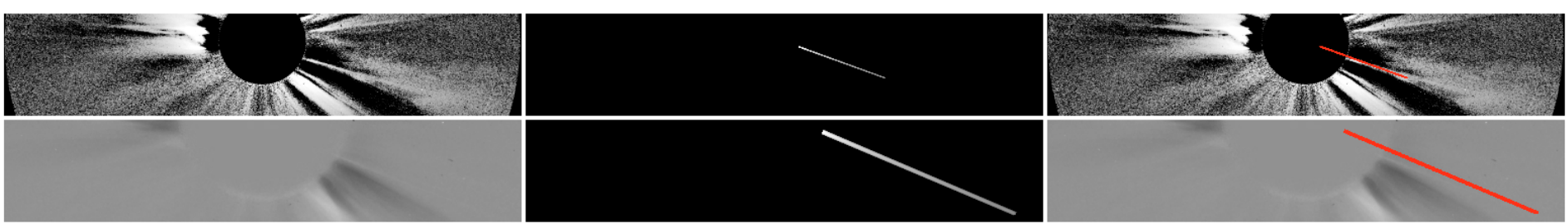

Fig. 7. 3D geometrical modeling of the ray observations of STA/COR2 (upper panel) and of LASCO/C2 (lower panel). The left column shows TB images for each spacecraft, where a pre-event image was first subtracted. The middle column shows TB images of the ray from our 3D modeling whereas the right column shows overlays of the rectangular slab model wireframe(red) on the corresponding TB images. Times of the observation: 17:52:20 UT for STA/COR2 and 17:50:04 for LASCO/C2.
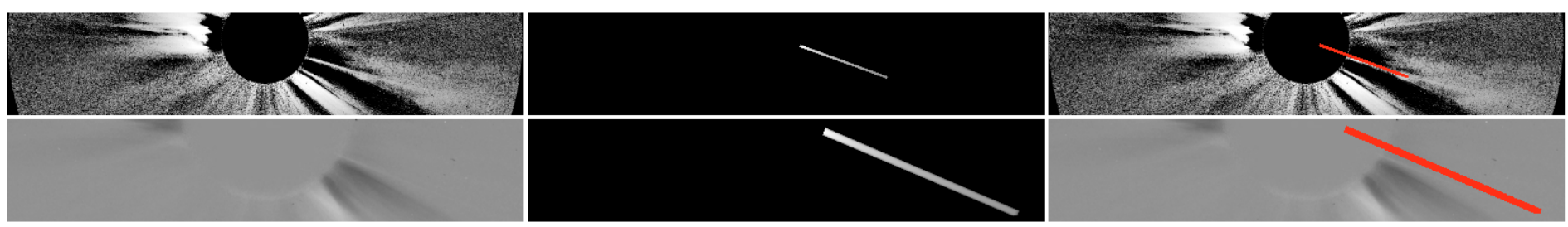

Fig. 8. Same as in Fig. 7 but for a ray model with twice the depth and the thickness of that of Fig. 7.

distance of the ray from the STA POS $\left(\approx 15^{\circ}\right)$. Since the ray orientation is degenerate the LOS extent of the ray can be in principle anywhere between the calculated ray thickness $\left(0.05 R_{\odot}\right)$ and depth $\left(0.15 R_{\odot}\right)$. We make the assumption it is $0.10 R_{\odot}$, i.e. the median value of the above interval. Therefore, the LOS extent uncertainty is $\pm 0.05 R_{\odot}$, which translates into $50 \%$ density uncertainty. In such a way we were able to determine "true" densities.

The determination of the ray densities involved the following steps. First, we created an excess mass image of the STA ray for the TB observation 18:07:54 UT UT by subtracting a pre-event image (taken at 09:22:54 UT). Both images were fully calibrated. The conversion from Mean Solar Brightness to electrons $/ \mathrm{cm}^{-2}$ was done following the standard assumptions of Thompson scattering (e.g., Vourlidas et al. 2000, for details) and the distance of the ray from the STA POS.

The excess mass image is shown in Fig. 9. White (black) regions represent places with excess (deficit) mass with respect to the pre-event reference image. Several points along the ray were then manually selected (dashed line in Fig. 9). To enhance the ray signal the mass measurements along the ray were binned every $0.02 R_{\odot}$. Then the excess mass of the ray was converted into a density by using its LOS extent from our 3D modeling. We note here that the derived densities are likely lower limits to the true densities. If the CME removes significant part of the overlying streamer, then the pre-event subtraction will result in lower excess brightness than expected. This is insignificant in our case, since there is only a diffuse preexisting structure at the location of our ray and the observed depletions are minimal at the ray location (especially on the northward side). Uncertainties due to photon counting statistics can be safely ignored given the very large count rates we have in WL at the heights of our observations (typically above thousand counts per pixel). The uncertainty in the excess mass measurements of the COR2 instruments is estimated to be $20 \%$. The total uncertainty arises from the quadratic combination of these uncertainties (mass determination and LOS depth) under the assumption they are independent (i.e., we took the average of the quadratic sum of the individual uncertainties).

The resulting density profile along the ray is given in Fig. 10 . Note also that the distances along the ray are also "true" in the sense we used our 3D CS modeling to convert POS distances

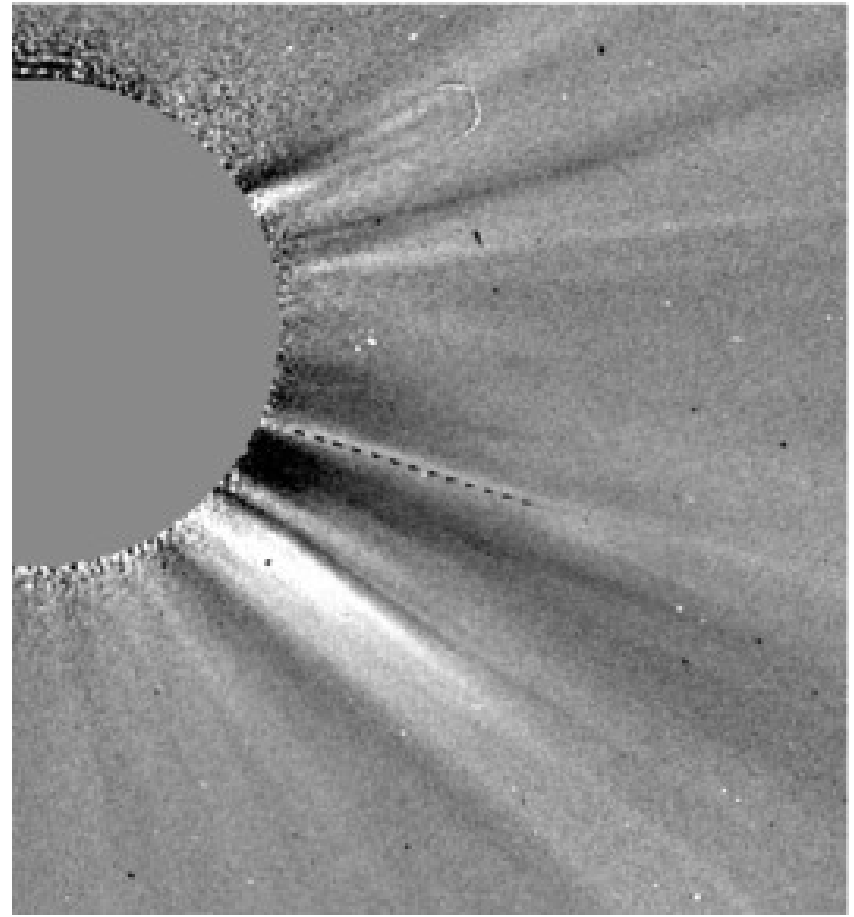

Fig. 9. Determining excess masses (densities) along the ray (dashed line) from the COR2A observation at 17:52:20 UT. A pre-event frame taken at 09:22:00 UT was subtracted.

to heliocentric distances. We plot densities only in the range $3.2-3.8 R_{\odot}$ because the ray becomes too diffuse at larger distances to be discerned from its surroundings. Several remarks can now be made. First, the derived density values are more than two orders of magnitude larger than the densities of the pre-CME corona at the heights of our observations. This can be seen in Fig. 10 where we plot densities from the inversion of the LASCO C2 PB sequence taken at 10:06 on the day of the event. The sequence was taken before the emergence of our CME into the $\mathrm{C} 2 \mathrm{FOV}$ and gives an estimate of the densities in the pre-CME corona. The pre-CME densities were calculated at the same position angle as the ray densities in Fig. 10. The deduced ray densities are within the range $\approx 10^{7}-10^{8} \mathrm{~cm}^{-3}$ determined for 


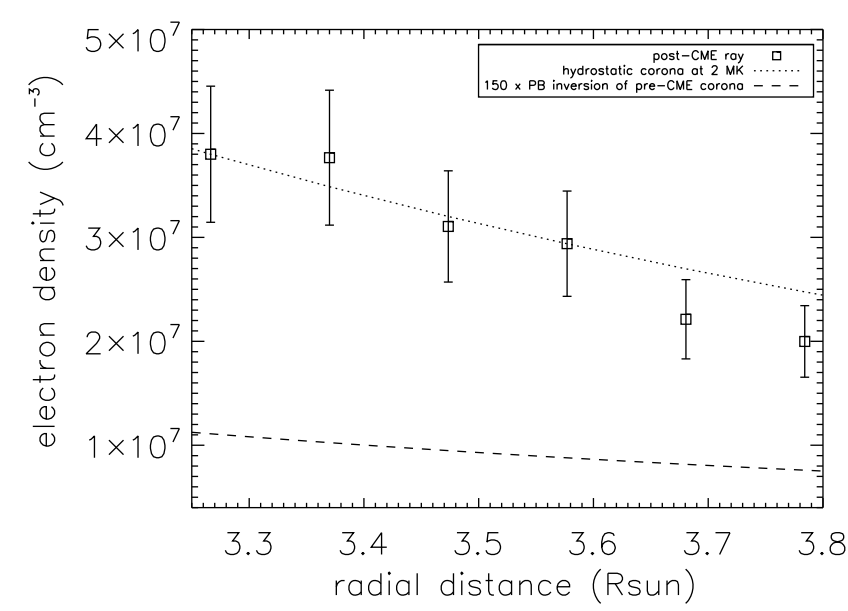

Fig. 10. Density along the ray as a function of heliocentric distance (squares). Density profile of the pre-CME corona on 9 April 2008, 10:06 UT from LASCO C2 PB data inversion (dashes). Density distributions for a hydrostatic isothermal corona at $2 \mathrm{MK}$ (dots).

several WL rays by Vršnak et al. (2009). Applying the same procedure to COR1 data, we found a ray density of $\approx 3 \times 10^{8} \mathrm{~cm}^{-3}$ at a distance of about $1.6 R_{\odot}$. Again the ray emission becomes too faint at larger heights to be reliably measured. Second, we note that the ray density does not substantially decrease with height along its length (only a factor $\approx 2$ over a distance of $\approx 0.5 R_{\odot}$ ). This implies reduced scales heights and therefore elevated temperatures within the ray. Indeed, we can see from Fig. 10 that the ray density drop-off can be fairly well reproduced by a hydrostatic density profile at a uniform temperature of $2 \mathrm{MK}$. In doing this we used a profile of the following form:

$n(h)=n_{0} \exp (-h / H)$,

where $h$ is the ray height above the solar surface, $n_{0}$ the density at the base of the ray and $H$ the isothermal scale-height (i.e., $\propto$ to temperature). Empirical models of the quiet corona indicate temperatures of $\leq 1 \mathrm{MK}$ at the heights of our observations (e.g., Withbroe 1988). We note here that the temperature is inferred under the assumption of a hydrostatic equilibrium. Such an assumption may not be fully justified for post-CME rays which are often characterized by dynamic phenomena such as plasma blobs.

\section{Discussion and conclusions}

This paper presents the first multi-viewpoint observations of a post-CME ray. Utilizing multiple view points together with detailed 3D geometrical modeling supplied new important insight into the 3D structure of a post-CME ray and its relationship with the associated CME. Our main findings are the following:

- The ray can be fitted by a rectangular slab with small $\left(\approx 0.15 R_{\odot}\right)$ depth and $\left(\approx 0.05 R_{\odot}\right)$ thickness.

- The ray axis is almost aligned with that of the CME.

- The ray lies within the CME volume and it occupies a much smaller volume than the CME.

- The ray is characterized by elevated $(>100)$ densities with respect to the ambient corona.

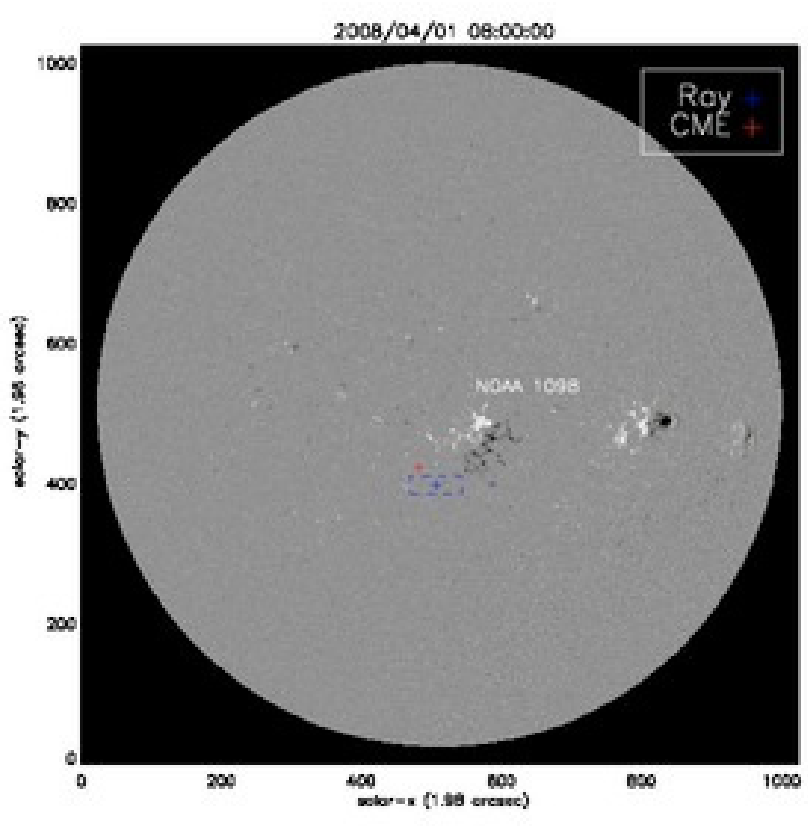

Fig. 11. Overlay of the CME (red cross) and ray (blue cross) central longitudes and latitudes determined from the 3D modeling of Sect. 3 on an MDI magnetogram taken during 1 April 2008, when the host AR (i.e., NOAA 1098) was close to the central meridian as viewed from SOHO. The dashed blue box has a length/height equal to the depth/thickness of the WL ray as determined from our 3D modeling. Its orientation cannot be determined from our modeling (see the discussion of Sect. 3).

- The ray density drop-off with height is consistent with a high $(\approx 2 \mathrm{MK})$ temperature, significantly hotter than the ambient corona at the same heights.

Our ray density and temperature findings are fully in line with previous post-CME ray observations with UVCS and LASCO which showed they are characterized by elevated densities and temperatures. It is reasonable to expect such behavior within CSs. The pinch-off of field lines resulting into a CS would compress the plasma thereby increasing its density. Also significant heating may occur in CSs since they are characterized by enhanced current densities.

Undoubtedly the most important implications of our study arise from the $3 \mathrm{D}$ analysis of the event. We have determined the 3D shape of a post-CME WL ray and deduced its width and depth. Figure 11 contains an overlay of the CME and the WL ray central longitudes and latitudes as determined from the 3D modeling of Sect. 3 on a MDI magnetogram taken on 1 April 2008, i.e. close to the central meridian passage of the host $A R$ as viewed from SOHO. We first note that both the CME (red cross) and WL ray (blue cross) are significantly misplaced towards the southwest direction with respect to the host AR. This suggests a non-radial evolution of the eruption in the inner corona and verifies the visual impressions of Sect. 2 (e.g., Fig. 1). Moreover, the $3 \mathrm{D}$ ray depth (equal to the vertical blue dashed lines of Fig. 11) is comparable to the length of the neutral line of the host AR. If the ray corresponds to a CS, we would expect its depth to be comparable to the length of the erupting neutral line as seems to be the case. However, the deduced ray width $\left(\approx 0.05 R_{\odot}\right)$ is still many orders of magnitude bigger than the expectations of standard plasma theory.

We also found that the CME and the ray were almost perfectly aligned. This supplies strong evidence for a tight and 

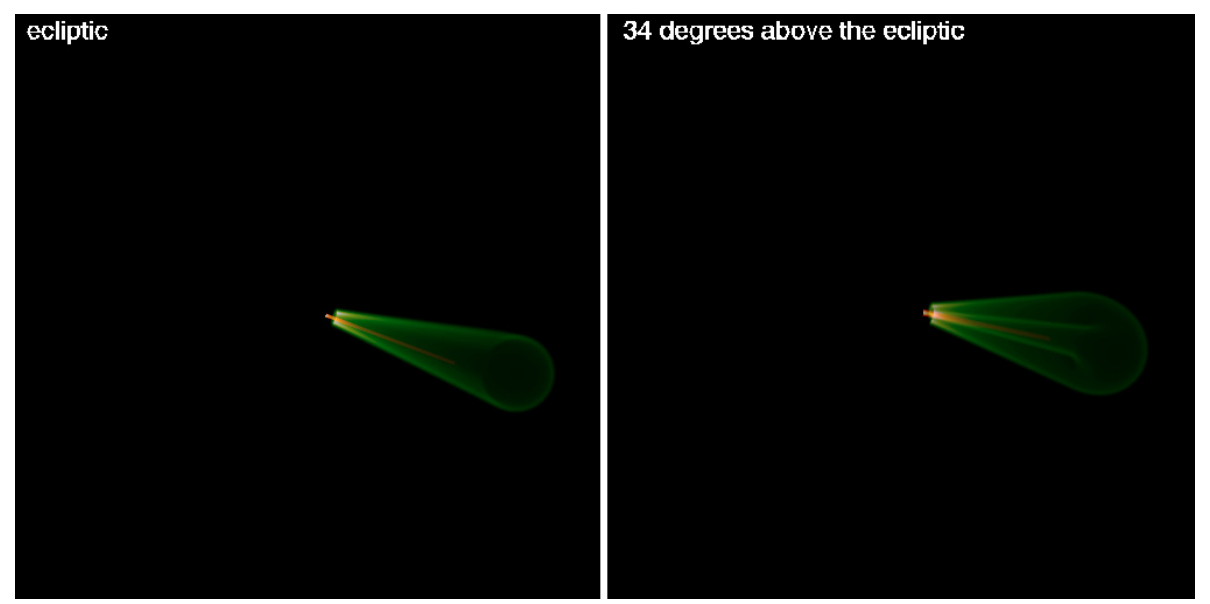

Fig. 12. Combined $\mathrm{TB}$ view of the $\mathrm{CME}$ (green) and of the ray (orange). View from the ecliptic plane (left panel). View 34 degrees above the ecliptic plane (right panel). Both views are from a distance of $100 R_{\odot}$.

causal relationship between CME expansion and ray(CS) formation. The ray(CS) should trail the CME if it results from the pinch off of magnetic field behind the eruption. The fact that the WL ray could represent a post-CME CS could help removing the degeneracy in the ray orientation discussed in Sect. 3 since they ray should lie in a plane perpedicular to the plane of $\mathrm{CME}$ expansion. The fact that the ray and CME are not perfectly aligned can be rather easily anticipated in terms of the various re-arrangements (rotations, deflections etc.) taking place during the event. Furthermore, according to the standard model for solar eruptions one expects that the post-eruption CS in the inner corona should lie above the post-flare loops which in turn are straddling the neutral line of the erupting AR. Since now the candidate CS (i.e., the WL ray) is misplaced with respect to the erupting neutral line suggests the possibility of a "broken" current sheet. This introduces a new, and possibly important, consideration for CME models.

As a further visualization of the intimate relationship between the ray and the CME Fig. 12 presents WL renderings of the ray and the CME from our modeling taken from two different views: one from the ecliptic plane and another $34^{\circ}$ above the ecliptic. The latter is a configuration which will be possible with the planned Solar Orbiter mission. What we note in this figure is that the ray is much smaller than the CME and it is located between its inner legs. It seems therefore likely according to the standard model for solar eruptions that once the CME leaves the FOV its legs will pinch off and give rise to a ray (CS).

In summarizing, our study clearly shows that the ray lies where it should be (aligned with the CME) and it is as big as it should be (depth similar to the erupting active region neutral line) if it had been a CS. Therefore, all our 3D findings give strong support to the possibility that the observed post-CME ray is related to a post-CME CS.

Acknowledgements. The SECCHI data used here were produced by an international consortium of the Naval Research Laboratory (USA), Lockheed Martin Solar and Astrophysics Lab (USA), NASA Goddard Space Flight Center (USA), Rutherford Appleton Laboratory (UK), University of Birmingham (UK), Max-Planck-Institut for Solar System Research (Germany), Centre Spatiale de Liège (Belgium), Institut d'Optique Théorique et Appliqueé (France), and Institut d'Astrophysique Spatiale (France). We thank the referee for useful comments which substancially impooved the paper.

\section{References}

Asai, A., Yokoyama, T., Shimojo, M., \& Shibata, K. 2004, ApJ, 605, L77 Auchère, F., Soubrié, E., Bocchialini, K., \& Legall, F. 2008, SoPh, 248, 213 Bemporad, A. 2008, ApJ, 689, 572

Bemporad, A., Poletto, G., Suess, S. T., et al. 2006, ApJ, 638, 1110

Billings, D. E. 1966, A Guide to the solar corona (New York: Academic Press) Brueckner, G. E., Howard, R. A., Koomen, M. J., et al. 1995, SoPh, 162, 357

Cargill, P. J., Vlahos, L., Turkmani, R., Galsgaard, K., \& Isliker, H. 2006, SSRv, 124, 249

Colaninno, R., \& Vourlidas, A. 2009, ApJ, 698, 852

Forbes, T. G. 2000, J. Geophys. Res., 105, 23153

Forbes, T. G., \& Lin, J. 2000, Journal of Atmospheric and Solar-Terrestrial Phys., 62,1499

Howard, R. A., Moses, J. D., Vourlidas, A., et al. 2008, Space Sci. Rev., 136, 67 Innes, D. E., McKenzie, D. E., \& Wang, T. 2003, SoPh, 217, 267

Kaiser, M. L., Kucera, T. A., Davila, J. M., et al. 2008, Space Sci. Rev., 136, 5 Klimchuk, J. A. 1996, in Magnetic Reconnection in the Solar Atmosphere, ed. R. Bentley, \& J. Mariska (San Francisco: Ast. Soc. Pacific), ASP Conf. Ser., 111,319

Ko, Y.-K., Raymond, J. C., Lin, J., et al. 2003, ApJ, 594, 1068

Ko, Y.-K., Raymond, J. C., Vrsnak, B., \& Vujic, E. 2010, ApJ, 722, 625

Kohl, J. L., Esser, R., Gardner, L. D., et al. 1995, Sol. Phys., 162, 313

Landi, E., Raymond, J. C., Miralles, M. P., \& Hara, H. 2010, ApJ, 711, 75

Lee, J.-Y., Raymond, J. C., Ko, Y.-K., \& Kim, K.-S. 2006, ApJ, 651, 566

Lin, J., \& Forbes, T. G. 2000, JGR, 105, 2375

Lin, J., Li, J., Forbes, T. G., et al. 2007, ApJ, 658, L123

Lin, J., Li, J., Ko, Y.-K., \& Raymond, J. C. 2009, ApJ, 693, 1666

Linton, M. G., \& Longcope, D. W. 2006, ApJ, 642, 1177

Litvinenko, Y. E. 1996, ApJ, 462, 997

McKenzie, D. E., \& Hudson, H. S. 1999, ApJ, 519, L93

Onofri, M., Isliker, H., \& Vlahos, L. 2006, Phys. Rev. Lett., 96, 151102

Poland, A. I., Howard, R. A., Koomen, M. J., Michels, D. J., \& Sheeley, N. R., Jr. 1981, SoPh, 69, 169

Raymond, J. C., Ciaravella, A., Dobrzycka, D., et al. 2003, ApJ, 597, 1106

Reeves, K. K., Patsourakos, S., Stenborg, G., et al. 2008, AGUFMSH12, A4 Savage, S. L., McKenzie, D. E., Reeves, K. K., Forbes, T. G., \& Longcope, D. W. 2010, ApJ, 722, 329

Sheeley, N. R., Jr., \& Wang, Y.-M. 2007, ApJ, 655, 1142

Sheeley, N. R., Jr., Warren, H. P., \& Wang, Y.-M. 2004, ApJ, 616, 1224

Thernisien, A. F. R., Howard, R. A., \& Vourlidas, A. 2006, ApJ, 652, 763

Thernisien, A., Vourlidas, A., \& Howard, R. A. 2009, Sol. Phys., 256, 111

Vršnak, B., Poletto, G., Vujic, E., et al. 2009, A\&A, 499, 905

Vourlidas, A., \& Howard, R. A. 2006, ApJ, 642, 1216

Vourlidas, A., Subramanian, P., Dere, K. P., \& Howard, R. A. 2000, ApJ, 534, 456

Webb, D. F., Burkepile, J., Forbes, T. G., \& Riley, P. 2003, JGRA, 108, 1440

Withbroe, G. L. 1988, ApJ, 325, 442

Wood, P., \& Neukirch, T. 2005, Sol. Phys., 226, 73 